\title{
Emergência e caracterização biométrica inicial de cajuzinho-arbóreo- do-cerrado (Anacardium othonianum Rizz.) em diferentes temperaturas e substratos
}

\section{Emergence and initial biometric characterization of Cerrado tree cashew (Anacardium othonianum Rizz.) at different temperatures}

\section{and substrates}

Cerrado tree cashew (Anacardium othonianum Rizz) is predominantly harvested, making it essential to master seedling production techniques for conservation and large-scale production. Thus, the objective of this research was to evaluate emergence and development of cerrado tree cashew at different temperatures and substrates. Fruits and pseudo fruits were collected in the region of Luziânia (GO). The experiment was carried out at the Biotechnolog laboratory of the Federal Goiano Institute - Campus Urutaí, where the emergence and development of cerrado tree cashew seedlings under different substrates and temperatures under controlled conditions were evaluated. The design was completely randomized in a $4 \times 4$ factorial scheme (four substrates $x$ four temperatures) and twenty replications. The substrates being: $S 1$ : commercial substrate + vermiculite (2: 1); S2: ravine earth + vermiculite

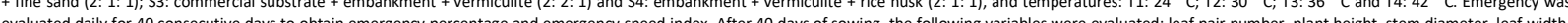
evaluated dally for 40 consective days to obtane + vermiculite + rice straw) and the temperature of $30^{\circ} \mathrm{C}$ presented the best conditions of emergence and in the development of cashew tree seedlings.

Keywords: Native fruits of the Cerrado; B.O.D; Seedling production.

Topic: Uso de Recursos Naturais

Reviewed anonymously in the process of blind peer.
Received: 25/06/2019

Approved: 26/07/2019
Ana Flávia de Jesus Pinto (D)

Instituto Federal Goiano, Brasil

http://lattes.cnpq.br/6338082904943098

http://orcid.org/0000-0001-9539-0344

anaflaviah15@gmail.com

Mateus Teixeira Rincon Cardoso (iD)

Instituto Federal Goiano, Brasil

http://lattes.cnpq.br/4923404893946856

http://orcid.org/0000-0002-4705-2284

mtrcardoso@hotmail.com

Cinthia Luzia Teixeira Silva (iD

Universidade Estadual de Goiás, Brasil

http://lattes.cnpq.br/9036428344570368

http://orcid.org/0000-0003-0740-1301

cinthiateixeirasilva@gmail.com

\begin{tabular}{l} 
Wellington José Pereira \\
Instituto Federal Goiano, Brasil \\
http://lattes.cnpq.br/3768969688629850 \\
\hline http://orcid.org/0000-0002-5897-636X \\
wellingtonjpereira74@gmail.com \\
Carla Geovanna Caixeta Issa (D) \\
Instituto Federal Goiano, Brasil \\
http://lattes.cnpq.br/9048424130070161 \\
http://orcid.org/0000-0001-7431-2160 \\
carlaisssa@gmail.com \\
Eli Regina Barboza de Souza (iD \\
Universidade Federal de Goiás, Brasil \\
http://lattes.cnpq.br/7641431808466123 \\
http://orcid.org/0000-0001-6225-6122 \\
eliregina1@gmail.com
\end{tabular}

Muza do Carmo Vieira

Instituto Federal Goiano, Brasil

http://lattes.cnpq.br/3892400622755069

http://orcid.org/0000-0003-1730-9541

mcvmuza@gmail.com

Ana Paula Silva Siqueira (id)

Instituto Federal Goiano, Brasil

http://lattes.cnpq.br/4670559561277136

http://orcid.org/0000-0002-4727-4127

ana.siqueira@ifgoiano.edu.br d

DOI: 10.6008/CBPC2179-6858.2019.004.0023
Referencing this:

PINTO, A. F. J.; CARDOSO, M. T. R.; SILVA, C. L. T.; PEREIRA, W. J.; ISSA, C. G. C.; SOUZA, E. R. B.; VIEIRA, M. C.; SIQUEIRA, A. P. S.. Emergência e caracterização biométrica inicial de cajuzinho-arbóreo-do-cerrado (Anacardium othonianum Rizz.) em diferentes temperaturas e substratos. Revista Ibero-Americana de Ciências Ambientais, v.10, n.4, p.301-313, 2019. DOI: http://doi.org/10.6008/CBPC2179$\underline{6858.2019 .004 .0023}$ 


\section{INTRODUÇÃO}

O Bioma Cerrado é um vasto ecossistema caracterizado por um gradiente de pastagens para savanas e formações florestais e pela grande riqueza de espécies (RIBEIRO et al., 2011). O Cerrado brasileiro é o segundo bioma mais extenso da América do Sul (SANO et al., 2010; BEUCHLE et al., 2015), ocupando uma área de 2 milhões de $\mathrm{km}^{2}$, representando 22\% da área total do Brasil (OLIVEIRA et al., 2014) distribuídos nos estados da Bahia, Goiás, Tocantins, Minas Gerais, Maranhão, Piauí, Mato Grosso, Mato Grosso do Sul, São Paulo e Paraná (SANO et al., 2010).

Com ampla variabilidade de espécies, o Cerrado apresenta utilidade em diversas áreas como agroindustrial, alimentar, energético, forrageiro, madeireiro, medicinal, ornamental e outros. As frutíferas nativas são espécies fundamentais neste ecossistema e há muitos anos são regularmente consumidas pelas populações locais tanto na forma in natura quanto como produtos processados, tais como sucos, sorvetes, pães e bolos (DAMIANI et al., 2011; MORZELLE et al., 2015). Entretanto, um vasto número de espécies frutíferas nativas e exóticas permanece inexplorado, apesar do alto potencial nutricional e econômico (SCHIASSI et al., 2018).

Entre as frutas nativas do Cerrado está o cajuzinho-arbóreo-do-cerrado (Anacardium othonianum Rizz). Pertencente à família Anacardiaceae, é uma frutífera nativa comumente conhecida como caju de árvore do cerrado (cajuzinho-arbóreo-do-cerrado), cajuzinho e cajuí (BESSA et al., 2013). As plantas adultas distinguem-se das demais espécies do gênero nesse bioma devido seu porte arbóreo. Sua exploração ocorre de forma extrativista e muitas vezes de modo predatório (ASSIS et al., 2012).

O cajuzinho-arbóreo-do-cerrado possui duas partes: o fruto verdadeiro e o pseudofruto (ALVES et al., 2013). O fruto é uma drupa reniforme (castanha), que contém cerca de $46,5 \%$ de lipídios (TOSCHI et al., 1993; CAETANO, et al., 2012). Os frutos são torrados para extrair as nozes, e possuem alto valor nutricional e energético e são ricos em proteínas, lipídios, cálcio, ferro e zinco (SILVA et al., 2017). O pseudofruto varia em tamanho e cor, podendo ser consumido fresco ou processado em doces, geleias, licores, sucos, sorvetes, picolés, frutas cristalizadas e outros produtos (DORNELLES et al., 2014).

A propagação de espécies vegetais é agrupada em dois tipos: a propagação sexuada e assexuada (FACHINELLE et al., 2005). Para a cultura do caju Agostini-Costa et al. (2006) relata que o processo de propagação vegetativa mais usado para o cajueiro é a enxertia por garfagem em fenda lateral ou por borbulha em placa. Enquanto a propagação por sementes é o método mais aplicado, proporcionando maior resistência das plântulas, que se desenvolvem com maior vigor e apresentando raízes até 20 vezes mais profundas, porém, são plantas que apresentarão frutificação tardia, porte elevado e irregularidade de produção (FACHINELLO et al., 1995).

O processo germinativo é regulado por vários fatores ambientais, como umidade, oxigênio, temperatura, luz e nutrientes (SEO et al., 2009; DOUSSEAU et al., 2011). E dentre os fatores do ambiente que afetam o processo germinativo das sementes, observa-se a existência de uma estreita dependência da temperatura (ARAÚJO-NETO et al., 2003). A temperatura influencia tanto na porcentagem de germinação 
Emergência e caracterização biométrica inicial de cajuzinho-arbóreo-do-cerrado (Anacardium othonianum Rizz.) em diferentes temperaturas e substratos

PINTO, A. F. J.; CARDOSO, M. T. R.; SILVA, C. L. T.; PEREIRA, W. J.; ISSA, C. G. C.; SOUZA, E. R. B.; VIEIRA, M. C.; SIQUEIRA, A. P. S.

quanto na determinação do vigor das plântulas, influenciando a absorção de água pela semente e as reações bioquímicas que regulam todo o processo metabólico (BEWLEY et al., 1994; PACHECO et al., 2006).

Para as plantas nativas como o cajuzinho-arbóreo-do-cerrado, tem-se poucas informações sobre a sua propagação, além disso, é importante saber em qual tipo de substrato ocorre o melhor desenvolvimento inicial das plântulas (SILVA et al., 2018). Um substrato deve atender aos requisitos da planta em termos de características físicas e químicas, pois substitui o solo durante o estágio de viveiro (FERRAZ et al., 2005; DORNELLES et al., 2014). Formulado por diversos materiais como matéria-prima de origem mineral, sintética ou orgânica, o substrato pode ser constituído por apenas um tipo deles, ou criando uma formulação entre os diferentes materiais (KANASHIRO, 1999).

No sentido de elucidar as características da produção de mudas de espécies de frutíferas nativas, quando se refere a temperatura e substrato algumas pesquisas tem sido realizadas, como as de Silva et al. (2009), Aparecida-Dias et al. (2011), Dousseau et al. (2011), Dresch et al. (2012) e Gomes et al. (2016), mas associados ao cajuzinho-arbóreo-do-cerrado são poucos estudos. Contudo, trabalhos como o de Silva et al. (2018) se destacam, esses pesquisadores avaliaram o desenvolvimento inicial do $A$. othonianum em diferentes substratos e combinações (substrato comercial, areia, esterco bovino, solo e casca de arroz) e concluíram que o melhor substrato para a propagação do cajuzinho foi composto por areia e substrato comercial. Todavia, é importante dominar as técnicas de produção de mudas, quanto a avaliação de diferentes substratos associadas temperaturas, quando trabalhado com frutíferas nativas do Cerrado, e em especial, cajuzinho-arbóreo-do-cerrado.

Diante das adversidades climáticas, da necessidade de estudos e preservação do patrimônio genético e produção mudas de qualidade, é de extrema importância ter em mãos informações sobre produção de mudas de frutíferas nativas do Cerrado. Assim, objetivou-se com este estudo avaliar emergência e desenvolvimento de cajuzinho-arbóreo-do-cerrado em diferentes temperaturas e substratos.

\section{METODOLOGIA}

Foi realizada uma viagem de prospecção para identificação e escolha de plantas de cajuzinhoarbóreo-do-cerrado para a coleta de frutos em uma propriedade rural (Latitude: 16 $57^{\prime} 30,27^{\prime \prime}$ S; Longitude: 4757'3,10" W; Altitude: 859m) no município de Luziânia, no estado de Goiás, Brasil. O trabalho foi conduzido no laboratório de biotecnologia do Instituto Federal Goiano Campus Urutaí (LABIOTEC), na cidade de Urutaí, Goiás.

Foram selecionadas plantas em áreas de formação natural de Cerrado, bem como aquelas que se encontravam isoladas em áreas de pastagens. Próximo as plantas matrizes, os frutos foram coletados manualmente sobre o solo ou coletados da planta, em seguida foram acondicionados em embalagens utilizadas para o transporte de ovos. As plantas matrizes das quais foram coletados os frutos, foram selecionadas levando em consideração aspectos fitossanitários externos e produção uniforme.

As sementes de cajuzinho-arbóreo-do-cerrado passaram por um processo de seleção, por teste de 
Emergência e caracterização biométrica inicial de cajuzinho-arbóreo-do-cerrado (Anacardium othonianum Rizz.) em diferentes temperaturas e substratos

PINTO, A. F. J.; CARDOSO, M. T. R.; SILVA, C. L. T.; PEREIRA, W. J.; ISSA, C. G. C.; SOUZA, E. R. B.; VIEIRA, M. C.; SIQUEIRA, A. P. S.

densidade, no qual foram colocadas em um béquer com água e aquelas que flutuaram, ficando na superfície foram descartadas, já aquelas que permaneceram ao fundo do béquer, foram utilizadas nos experimentos.

Avaliou-se a emergência e o desenvolvimento do cajuzinho-arbóreo-do-cerrado em diferentes substratos e temperaturas em condições controladas de laboratório. $\mathrm{O}$ delineamento adotado foi o inteiramente casualizado em esquema fatorial $4 \times 4$ (quatro substratos $x$ quatro temperaturas) e vinte repetições. Sendo os substratos: S1: substrato comercial + vermiculita (2:1); S2: terra de barranco + vermiculita + areia fina (2:1:1); S3: substrato comercial + terra de barranco + vermiculita (2:2:1) e S4: terra de barranco + vermiculita + casca de arroz(2:1:1). Todos os substratos receberam 4,20kg por $\mathrm{m}^{3}$ de substrato da formulação 05-25-15. O experimento foi conduzido nas temperaturas de $\mathrm{T} 1: 24^{\circ} \mathrm{C} ; \mathrm{T} 2: 30^{\circ} \mathrm{C} ; \mathrm{T} 3: 36^{\circ} \mathrm{C}$ e T4: $42^{\circ} \mathrm{C}$.

O experimento foi instalado em copos plásticos descartáveis de $200 \mathrm{ml}$ com o fundo perfurado e dispostos em câmara de demanda de oxigênio (B.O.D.). Em cada copo foi colocada uma semente. Cada tratamento foi mantido por 40 dias na B.O.D., com exposição a luz de 12 horas por dia, foram realizadas irrigação diariamente conforme a necessidade.

Após a instalação do experimento foram determinados o Índice de Velocidade de Emergência (IVE) e a porcentagem de emergência (E\%), registrando-se diariamente o número de plântulas emergidas, levando em consideração a emergência de uma estrutura em forma de 'joelho', culminado com a abertura completa dos cotilédones. Ocorrendo a estabilização da emergência, realizou-se o cálculo do índice de velocidade de emergência de acordo com Maguire (1962): IVE = E1/N1 + E2/N2 + ... En/Nn, onde: IVE = índice de velocidade de emergência; E1, E2, En = Número de plântulas emergidas computadas na primeira contagem, segunda contagem e na última contagem e N1, N2, Nn = Número de dias de semeadura à primeira, segunda e última contagem.

Enquanto para o cálculo da porcentagem de emergência adotou-se a metodologia de Labouriau et al. (1976): $E \%$ = (N/A).100. Onde: $E \%$ = Porcentagem de emergência; $N$ = Número de plântulas emergidas e $A$ = Número total de sementes colocadas para germinar. Ao completar 40 dias após a semeadura (DAS) foram selecionadas 5 plantas de cada tratamento de maneira aleatória e foram analisadas as seguintes variáveis: número de par de folhas (NPF), altura da planta (AP) (cm), diâmetro do caule (DC) (mm), largura da folha (DF) $(\mathrm{cm})$, comprimento da folha $(\mathrm{CF})(\mathrm{cm})$, comprimento da raiz $(\mathrm{CR})(\mathrm{cm})$, matéria fresca $(\mathrm{MF})(\mathrm{g})$ e matéria seca (MS) (g).

A altura de planta, comprimento da maior raiz e o comprimento da folha foram medidos utilizando uma régua graduada e com o auxílio de um paquímetro digital foi possível determinar o diâmetro do caule e o diâmetro da folha. Após a determinação da matéria fresca as plantas foram colocadas no interior de envelopes de papel previamente identificados, e levados a estufa a $70^{\circ} \mathrm{C}$ por 72 horas para a determinação da matéria seca. Para a determinação das matérias fresca e seca utilizou-se uma balança analítica. Os dados obtidos nos experimentos foram submetidos à análise de variância e as médias dos tratamentos foram comparadas pelos testes de Tukey e análise de regressão a 5\% de probabilidade com o auxílio dos softwares 
Sisvar (FERREIRA, 2000) e Assistat 7.7 Beta.

\section{RESULTADOS}

As temperaturas e os substratos proporcionaram diferenças significativas para a porcentagem de emergência e o índice de velocidade de emergência (tabela 1). Dentre os substratos utilizados, o que apresentou o maior E\% e IVE (tabela 1) foi o substrato S4 na temperatura de $30^{\circ} \mathrm{C}(95 \%$ e 5,45 respectivamente).

Tabela 1: Percentual de emergência (E\%) e índice de velocidade de emergência (IVE) de sementes de cajuzinhoarbóreo-do-cerrado (Anacardium othonianum Rizz.), submetidas a diferentes temperaturas e substratos. Urutaí (GO).

\begin{tabular}{|c|c|c|c|c|c|}
\hline \multirow[b]{2}{*}{ Substrato } & \multicolumn{4}{|c|}{ Temperatura } & \multirow{3}{*}{ CV\% } \\
\hline & $24^{\circ} \mathrm{C}$ & $30^{\circ} \mathrm{C}$ & $36^{\circ} \mathrm{C}$ & $42^{\circ} \mathrm{C}$ & \\
\hline & \multicolumn{4}{|c|}{$\mathrm{E} \%$} & \\
\hline S1 & $25 \mathrm{bA}^{1}$ & $40 \mathrm{bA}$ & $35 \mathrm{bA}$ & $25 a A$ & 64,66 \\
\hline S2 & 50abA & 70abA & 70abA & $40 a A$ & 30,12 \\
\hline S3 & $40 a b A B$ & 70abA & $50 a b A B$ & $30 a B$ & 32,13 \\
\hline S4 & $65 a B C$ & $95 a A$ & $75 a A B$ & $45 \mathrm{aC}$ & 14,29 \\
\hline \multirow[t]{2}{*}{ CV\% } & 37,41 & 24,84 & 30,95 & 34,99 & - \\
\hline & \multicolumn{4}{|c|}{ IVE } & \\
\hline S1 & $0,96 \mathrm{bB}$ & $1,69 \mathrm{cAB}$ & $1,56 \mathrm{bAB}$ & $1,35 \mathrm{aAB}$ & 49,18 \\
\hline S2 & $1,92 \mathrm{abA}$ & $3,83 a b A$ & $3,24 a b A$ & $2,38 \mathrm{aA}$ & 29,55 \\
\hline S3 & 1,43abA & $2,69 \mathrm{bcA}$ & $1,98 \mathrm{bA}$ & $1,74 a A$ & 36,83 \\
\hline S4 & $2,71 \mathrm{aC}$ & $5,45 a A$ & $3,91 \mathrm{aBC}$ & $2,93 \mathrm{aC}$ & 16,6 \\
\hline CV\% & 37,46 & 24,23 & 31,84 & 36,04 & - \\
\hline
\end{tabular}

Legenda: ${ }^{1}$ Médias seguidas por letras iguais não diferem entre si pelo teste de Tukey a $5 \%$ de probabilidade. Letras minúsculas representam análise nas colunas e maiúsculas nas linhas. CV (\%): Coeficiente de variação.

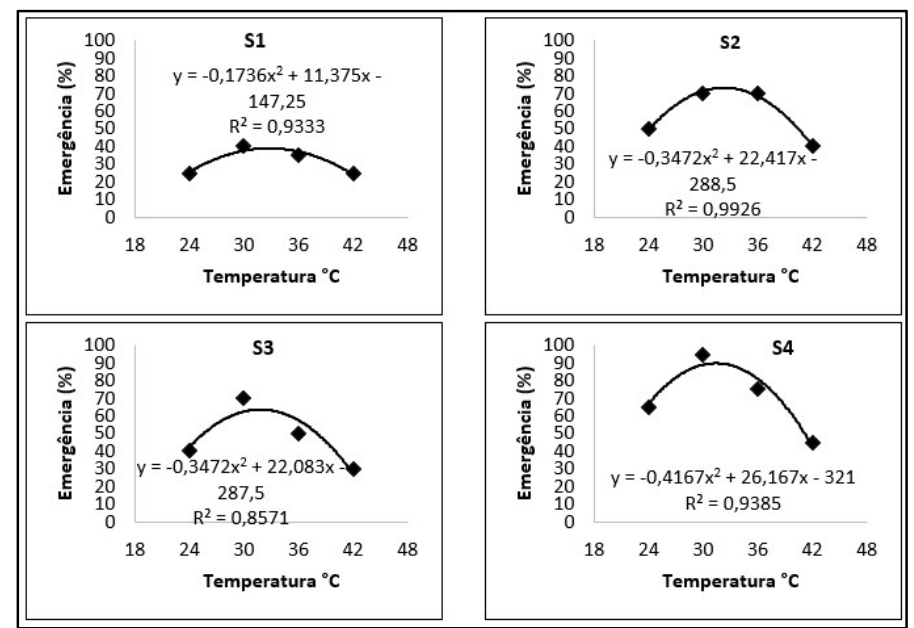

Figura 1: Percentagem de emergência de cajuzinho-arbóreo-do-cerrado (Anacardium othonianum Rizz.), em diferentes temperaturas e substratos. S1: substrato comercial + vermiculita (2:1); S2: terra de barranco + vermiculita + areia fina (2:1:1); S3: substrato comercial + terra de barranco + vermiculita (2:2:1) e S4: terra de barranco + vermiculita + casca de arroz (2:1:1). Urutaí (GO).

O substrato que apresentou menor E\% foi o S1 nas temperaturas de 24 e $42^{\circ} \mathrm{C}$. Enquanto, o substrato S1 associado a temperatura de $24^{\circ} \mathrm{C}$ apresentou o menor índice de velocidade de emergência (VE) $(0,96)$. Observou-se que a temperatura não interferiu significativamente na porcentagem de emergência nos substratos S1 e S2, e não interferiu no IVE dos substratos S2 e S3, apresentando-se significativa para os demais substratos. Verificou-se também que na temperatura de $42^{\circ} \mathrm{C}$, os diferentes substratos não se diferenciaram estatisticamente entre si. Independente do substrato utilizado (figura 1), na temperatura de $30^{\circ} \mathrm{C}$ (T2) ocorreu um porcentual de germinação superior as temperaturas 24 e $42^{\circ} \mathrm{C}$ (T1 e T4, 
respectivamente), se igualando ao tratamento com $36^{\circ} \mathrm{C}$. Foi constatada média geral de $68,75 \%$ de emergência entre as diferentes temperaturas.

Verificou-se que na temperatura de $36^{\circ} \mathrm{C}$ houve o maior número de par de folhas por planta cultivada nos diferentes substratos (tabela 2, figura 2). O substrato S4 apresentou o maior NPF nas temperaturas de $36^{\circ} \mathrm{C}$ e $42^{\circ} \mathrm{C}$ (4,33 e 4,22 pares, respectivamente), enquanto as plantas cultivadas no substrato $\mathrm{S} 1$ a $24^{\circ} \mathrm{C}$ (T1) apresentaram o menor NPF (2,80 pares). Entretanto, não houve diferenças significativas entre as plantas cultivadas nos diferentes substratos dentro das temperaturas de $24^{\circ} \mathrm{C}(\mathrm{T} 1), 36^{\circ} \mathrm{C}$ (T3) e $42^{\circ} \mathrm{C}$ (T4).

A maior altura de plantas (tabela 2) foi verificada nas plantas cultivadas no substrato S4 a uma temperatura de $42^{\circ} \mathrm{C}(9,48 \mathrm{~cm})$. Ao passo que, as plantas cultivadas no substrato $\mathrm{S} 3$ a $24^{\circ} \mathrm{C}$ apresentaram a menor AP $(2,70 \mathrm{~cm})$. As plantas cultivadas a $42^{\circ} \mathrm{C}$ (T4) nos diferentes substratos foram as que apresentaram as maiores alturas de plantas $(7,36$ a 9,48cm). A AP apresentou taxa de crescimento linear de acordo com a elevação da temperatura que se encontrava as mudas de cajuzinho-arbóreo-do-cerrado (figura 3).

Tabela 2: Número de par de folhas (Unidade), Altura de plantas $(\mathrm{cm})$, diâmetro do caule $(\mathrm{mm})$, largura da folha $(\mathrm{cm})$, comprimento de folhas $(\mathrm{cm})$ e comprimento da raiz $(\mathrm{cm})$ de cajuzinho-arbóreo-do-cerrado (Anacardium othonianum Rizz.) em diferentes temperaturas e substratos. Urutaí (GO).

\begin{tabular}{|c|c|c|c|c|c|}
\hline \multirow{3}{*}{ Substrato } & \multicolumn{4}{|c|}{ Temperatura } & \multirow{3}{*}{ CV\% } \\
\hline & \multicolumn{4}{|c|}{ Número de par de folhas } & \\
\hline & $24^{\circ} \mathrm{C}$ & $30^{\circ} \mathrm{C}$ & $36^{\circ} \mathrm{C}$ & $42^{\circ} \mathrm{C}$ & \\
\hline S1 & $2,80 \mathrm{aC}$ & $3,12 \mathrm{bBC}$ & $4,42 a A$ & $3,80 a A B$ & 15,25 \\
\hline $\mathrm{S} 2$ & $3,50 a B$ & $3,78 \mathrm{abAB}$ & $4,15 a A$ & $3,75 a A B$ & 14,18 \\
\hline S3 & $3,37 \mathrm{aB}$ & $3,50 a b A B$ & $4,10 a A$ & $3,83 a A B$ & 17,17 \\
\hline S4 & $3,46 a \mathrm{~B}$ & $3,94 a A B$ & $4,33 a A$ & $4,22 a A$ & 15,37 \\
\hline \multirow[t]{2}{*}{ CV\% } & 15,25 & 17,96 & 15,45 & 11,25 & - \\
\hline & \multicolumn{4}{|c|}{ Altura da planta } & \\
\hline S1 & $2,78 \mathrm{bC}$ & $5,18 \mathrm{bcB}$ & $5,83 \mathrm{bB}$ & $7,36 \mathrm{bA}$ & 12,86 \\
\hline $\mathrm{S} 2$ & $3,86 a b c$ & $5,95 a b B$ & $6,63 a B$ & $8,53 a b A$ & 14,03 \\
\hline S3 & $2,70 \mathrm{bD}$ & $4,95 \mathrm{cC}$ & $6,04 \mathrm{bB}$ & $8,14 \mathrm{bA}$ & 15,85 \\
\hline S4 & $4,86 \mathrm{aC}$ & $6,86 a \mathrm{~B}$ & $6,86 \mathrm{aB}$ & $9,48 \mathrm{aA}$ & 13,80 \\
\hline \multirow[t]{2}{*}{ CV\% } & 24,27 & 15,71 & 7,51 & 9,74 & - \\
\hline & \multicolumn{4}{|c|}{ Diâmetro do caule } & \\
\hline S1 & $2,96 \mathrm{bA}$ & $2,94 a A$ & $3,06 \mathrm{bA}$ & $2,70 a A$ & 13,74 \\
\hline $\mathrm{S} 2$ & $3,31 \mathrm{bA}$ & $3,15 a A$ & $3,56 a b A$ & $2,43 a B$ & 14,35 \\
\hline S3 & $3,18 \mathrm{bA}$ & $3,25 a A$ & $3,34 \mathrm{bA}$ & $2,60 a B$ & 12,18 \\
\hline S4 & $4,01 \mathrm{aA}$ & $3,40 \mathrm{aB}$ & $3,84 a A B$ & $2,45 \mathrm{aC}$ & 13,51 \\
\hline \multirow[t]{2}{*}{ CV\% } & 14,03 & 12,99 & 11,23 & 14,69 & - \\
\hline & \multicolumn{4}{|c|}{ Largura da folha } & \\
\hline S1 & $2,19 a \mathrm{~A}$ & $2,12 \mathrm{aA}$ & $1,73 a \mathrm{~A}$ & $1,72 \mathrm{aA}$ & 25,99 \\
\hline $\mathrm{S} 2$ & $2,27 a A B$ & $2,53 a A$ & $1,95 \mathrm{aB}$ & $1,94 \mathrm{aB}$ & 21,04 \\
\hline S3 & $2,04 a A$ & $2,13 a A$ & $1,85 a A$ & $1,75 a A$ & 24,46 \\
\hline S4 & $2,50 a A$ & $2,39 a A$ & $2,16 a A$ & $1,91 a A$ & 23,4 \\
\hline \multirow[t]{2}{*}{ CV\% } & 24,27 & 21,02 & 26,76 & 14,34 & - \\
\hline & \multicolumn{4}{|c|}{ Comprimento da folha } & \\
\hline S1 & $5,54 \mathrm{bAB}$ & $6,32 \mathrm{bcA}$ & $6,41 \mathrm{bA}$ & $4,97 \mathrm{bB}$ & 13,87 \\
\hline $\mathrm{S} 2$ & $6,12 \mathrm{bBC}$ & $7,00 a b A B$ & $7,16 a b A$ & $5,66 \mathrm{aC}$ & 12,42 \\
\hline S3 & $5,08 \mathrm{bB}$ & $6,88 \mathrm{bA}$ & $6,65 \mathrm{bAB}$ & $5,36 a b B$ & 17,86 \\
\hline S4 & $7,30 \mathrm{aA}$ & $7,88 \mathrm{aA}$ & $7,80 \mathrm{aA}$ & $5,62 \mathrm{aB}$ & 16,53 \\
\hline \multirow[t]{2}{*}{ CV\% } & 15,15 & 19,05 & 14,23 & 7,10 & - \\
\hline & \multicolumn{4}{|c|}{ Comprimento da raiz } & \\
\hline S1 & $5,74 \mathrm{bB}$ & $7,70 \mathrm{bAB}$ & $9,06 a \mathrm{~A}$ & $7,84 \mathrm{bAB}$ & 20,09 \\
\hline $\mathrm{S} 2$ & $7,58 a b C$ & $10,82 \mathrm{abAB}$ & $12,32 \mathrm{aA}$ & $8,95 a b B C$ & 11,89 \\
\hline S3 & $8,86 a A$ & $10,46 a b A$ & $11,70 \mathrm{aA}$ & $8,94 a b A$ & 20,94 \\
\hline S4 & $9,36 \mathrm{aA}$ & $13,64 a \mathrm{~A}$ & $11,84 \mathrm{aA}$ & $10,28 \mathrm{aA}$ & 25,94 \\
\hline CV\% & 16,23 & 27,58 & 17,11 & 13,94 & - \\
\hline
\end{tabular}

Legenda: ${ }^{1}$ Médias seguidas por letras iguais não diferem entre si pelo teste de Tukey a $5 \%$ de probabilidade. Letras minúsculas representam análise nas colunas e maiúsculas nas linhas. CV (\%): Coeficiente de variação. 


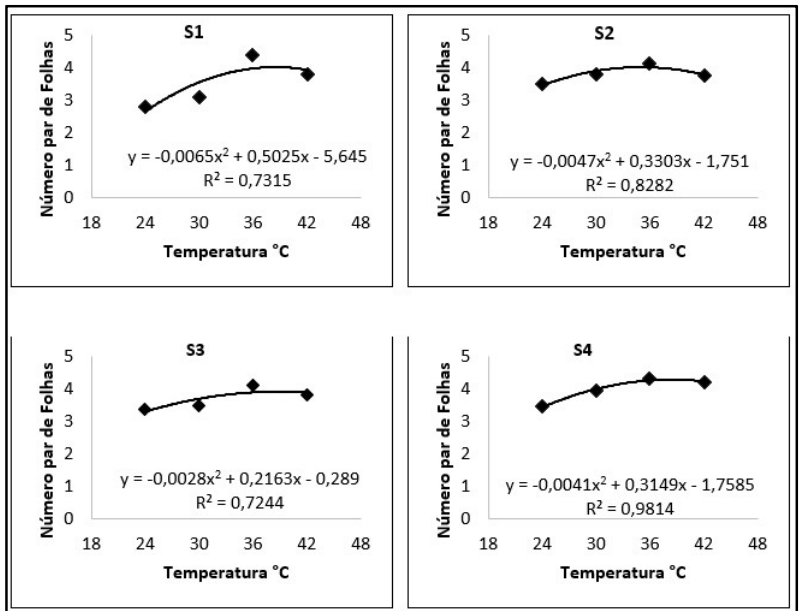

Figura 2: Número de par de folhas de mudas cajuzinho-arbóreo-do-cerrado (Anacardium othonianum Rizz.), em diferentes temperaturas e substratos. S1: substrato comercial + vermiculita (2:1); S2: terra de barranco + vermiculita + areia fina (2:1:1); S3: substrato comercial + terra de barranco + vermiculita (2:2:1) e S4: terra de barranco + vermiculita + casca de arroz(2:1:1). Urutaí (GO).

O diâmetro de caule das mudas cultivadas nos diferentes substratos diminuiu com o aumento da temperatura (tabela 2). Nas temperaturas de $24^{\circ} \mathrm{C}$ e $36^{\circ} \mathrm{C}$ o substrato $\mathrm{S} 4$ apresentou resultados superiores de diâmetro (4,01 e 3,84mm, respectivamente), diferenciando-se significativamente dos demais substratos. Entretanto, não houve diferenças significativas entre os substratos nas temperaturas de 30 e $42^{\circ} \mathrm{C}$, e entre as temperaturas no substrato S1.

A largura das folhas (DF) de caju-arbóreo-do-cerrado apresentou diferença significativa apenas entre as diferentes temperaturas no substrato $\mathrm{S} 2$ (tabela 2). As plantas cultivadas a $30^{\circ} \mathrm{C}$ neste mesmo substrato (figura 3) apresentaram valor superior aos demais do mesmo substrato $(2,53 \mathrm{~cm})$. Enquanto, não houve diferença significativa entre as diferentes temperaturas associadas aos outros substratos. As plantas cultivadas no substrato $\mathrm{S} 4$ foram as que apresentaram os maiores comprimento de folhas $(5,52$ a $7,88 \mathrm{~cm})$ independente da temperatura utilizada. As temperaturas de 30 e $36^{\circ} \mathrm{C}$ foram aquelas que apresentaram maior comprimento de folhas (figura 4), com linha de tendência quadrática referente a seus respectivos substratos associados.

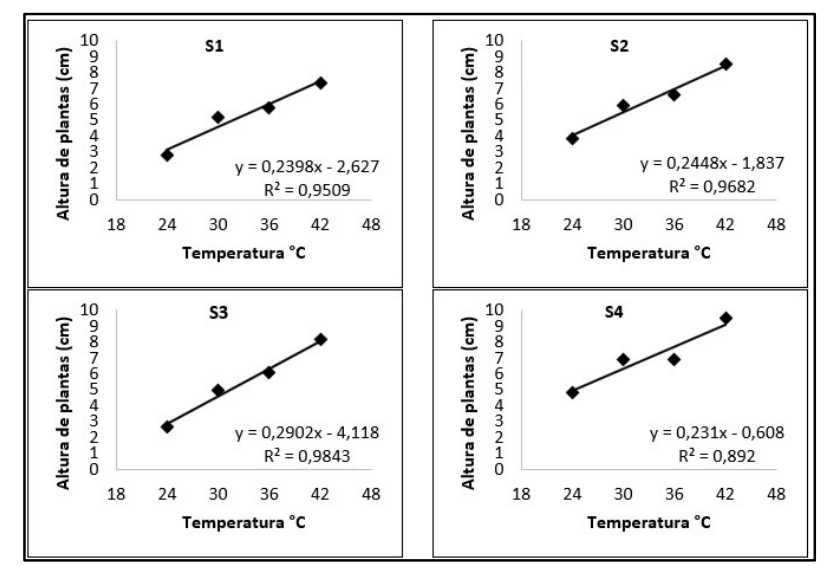

Figura 3: Altura de plantas de cajuzinho-arbóreo-do-cerrado (Anacardium othonianum Rizz.), em diferentes temperaturas e substratos. S1: substrato comercial + vermiculita (2:1); S2: terra de barranco + vermiculita + areia fina (2:1:1); S3: substrato comercial + terra de barranco + vermiculita (2:2:1) e S4: terra de barranco + vermiculita + casca de arroz (2:1:1). Urutaí (GO).

No comprimento da raiz (CR) os melhores resultados (tabela 2) foram obtidos pelos substratos S2, S3 
e S4. A utilização do substrato S1 apresentou resultados inferiores em todas as temperaturas, enquanto, o substrato S4 apresentou médias superiores independente da temperatura $(9,36$ a 13,64cm). No gráfico de progressão da temperatura, o CR apresentou linha de tendência quadrática em qualquer substrato (Figura $5)$, em que os substratos $S 1(9,06 \mathrm{~cm}), S 2(12,32 \mathrm{~cm})$ e $S 3(11,70 \mathrm{~cm})$ apresentaram as melhores médias na a $36^{\circ} \mathrm{C}$ e o substrato $\mathrm{S} 4(13,64 \mathrm{~cm})$ apresentou plantas com o maior comprimento de raiz a $30^{\circ} \mathrm{C}$.

Os substratos e diferentes temperaturas também proporcionaram diferenças significativas para as massas fresca e seca (tabela 3). Conforme o aumento da temperatura, as mudas de cajuzinho-arbóreo-docerrado cultivadas no substrato S2 apresentaram menor matéria fresca. Neste mesmo substrato foi observado que as plantas cultivadas a $24^{\circ} \mathrm{C}$ obtiveram a maior matéria fresca $(3,34 \mathrm{~g})$. Apenas no substrato S4, foi possível observar diferença significativa para a massa seca entre as temperaturas.

As mudas deste substrato na temperatura de $42^{\circ} \mathrm{C}$ evidenciou ter características iguais as mudas cultivadas a 24 e $30^{\circ} \mathrm{C}$, e inferior as mudas a $36^{\circ} \mathrm{C}$. Analisando os substratos, verificou-se diferença significativa apenas na temperatura de $24^{\circ} \mathrm{C}$, o substrato $\mathrm{S} 2$ apresentou as plantas com maior massa seca $(0,33 \mathrm{~g})$. Os demais substratos quando comparados com as diferentes temperaturas, não apresentaram diferença estatística entre eles.

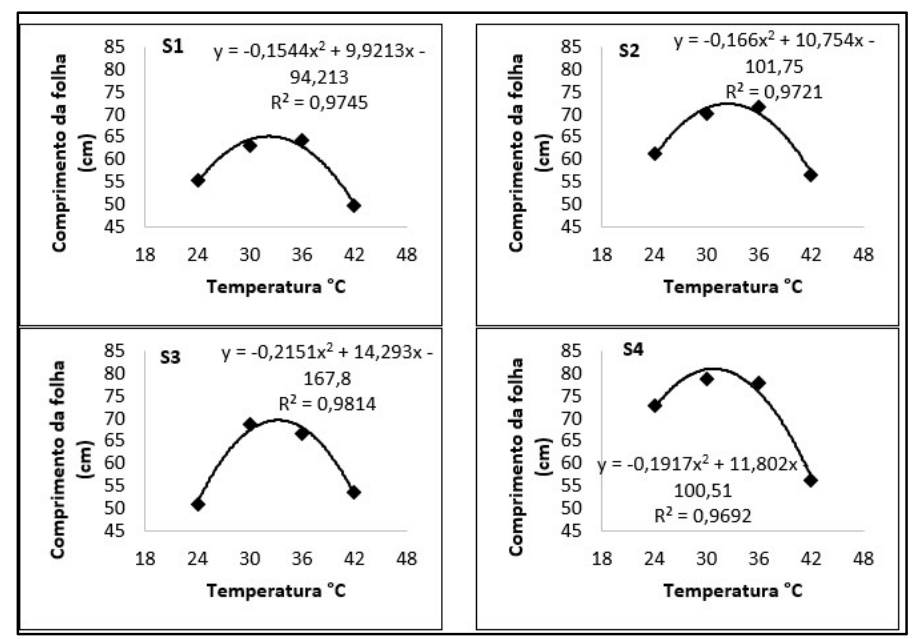

Figura 4: Comprimento da folha de mudas cajuzinho-arbóreo-do-cerrado (Anacardium othonianum Rizz.), em diferentes temperaturas e substratos. S1: substrato comercial + vermiculita (2:1); S2: terra de barranco + vermiculita + areia fina (2:1:1); S3: substrato comercial + terra de barranco + vermiculita (2:2:1) e S4: terra de barranco + vermiculita + casca de arroz (2:1:1). Urutaí (GO).

Tabela 3: Massa fresca (g) e massa seca (g) de cajuzinho-arbóreo-do-cerrado (Anacardium othonianum Rizz.) em diferentes temperaturas e substratos. Urutaí (GO).

\begin{tabular}{|c|c|c|c|c|c|}
\hline \multirow{3}{*}{ Substrato } & \multicolumn{4}{|c|}{ Temperatura } & \multirow{3}{*}{ CV\% } \\
\hline & $24^{\circ} \mathrm{C}$ & $30^{\circ} \mathrm{C}$ & $36^{\circ} \mathrm{C}$ & $42^{\circ} \mathrm{C}$ & \\
\hline & \multicolumn{4}{|c|}{ Massa fresca } & \\
\hline S1 & $2,02 \mathrm{cB}$ & $2,88 \mathrm{aA}$ & $2,04 \mathrm{bB}$ & $2,11 \mathrm{aB}$ & 15,83 \\
\hline S2 & $3,34 a b A$ & $2,76 a A B$ & $2,49 a A B$ & $2,12 \mathrm{aB}$ & 19,51 \\
\hline S3 & $1,93 c A$ & $2,48 a A$ & $2,00 \mathrm{bA}$ & $1,90 a A$ & 18,8 \\
\hline S4 & $2,58 \mathrm{bA}$ & $2,95 a A$ & $2,84 a A$ & $2,15 \mathrm{aA}$ & 16,69 \\
\hline \multirow[t]{2}{*}{ CV\% } & 12,3 & 23,31 & 17,59 & 11,37 & - \\
\hline & \multicolumn{4}{|c|}{ Massa seca } & \\
\hline S1 & $0,30 \mathrm{abA}$ & $0,27 a A$ & $0,31 a A$ & $0,23 a A$ & 16,47 \\
\hline S2 & $0,33 a A$ & $0,32 a A$ & $0,33 a A$ & $0,22 a A$ & 20,75 \\
\hline S3 & $0,26 \mathrm{bA}$ & $0,30 a A$ & $0,28 \mathrm{aA}$ & $0,22 a A$ & 15,28 \\
\hline S4 & $0,29 a b A B$ & $0,31 \mathrm{aAB}$ & $0,34 a A$ & $0,24 a B$ & 12,96 \\
\hline CV\% & 11,4 & 21,06 & 18,31 & 11,07 & - \\
\hline
\end{tabular}




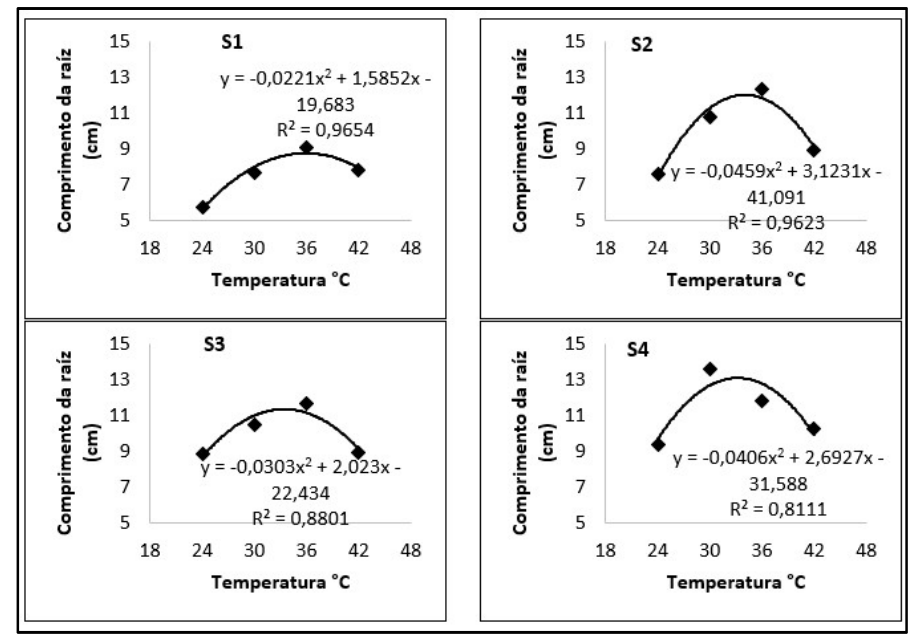

Figura 5: Comprimento da raiz de mudas cajuzinho-arbóreo-do-cerrado (Anacardium othonianum Rizz.), em diferentes temperaturas e substratos. S1: substrato comercial + vermiculita (2:1); S2: terra de barranco + vermiculita + areia fina (2:1:1); S3: substrato comercial + terra de barranco + vermiculita (2:2:1) e S4: terra de barranco + vermiculita + casca de arroz (2:1:1). Urutaí (GO). Legenda: ${ }^{1}$ Médias seguidas por letras iguais não diferem entre si pelo teste de Tukey a $5 \%$ de probabilidade. Letras minúsculas representam análise nas colunas e maiúsculas nas linhas. CV (\%): Coeficiente de variação.

\section{DISCUSSÃO}

Os maiores porcentuais de emergência e índice de velocidade de emergência foram obtidos a temperatura de $30^{\circ} \mathrm{C}$ (tabela 1; figura 1), resultados estes semelhantes aos encontrados por Oliveira et al. (2005) em Ipê Roxo, em que a emergência de plântulas mais adequada também foi em temperatura de $30^{\circ} \mathrm{C}$. Para a temperatura ser considerada como ótima ela deve apresentar as mais altas porcentagens de germinação e velocidade de germinação, na qual temperaturas acima e abaixo dela a germinação é prejudicada (MAYER et al., 1989; STOCKMAN et al., 2007).

As espécies vegetais apresentam uma grande variação quanto à temperatura ideal de germinação de suas sementes, no geral, esta faixa, está situada entre as temperaturas encontradas em sua região de origem e na época natural de emergência (ANDRADE et al., 2000). É importante ressaltar que o Cerrado está situado em região de clima tropical, é um tipo único de savana no mundo (SILVA et al., 2009). A temperatura média anual é de $22-28^{\circ} \mathrm{C}$ (LATRUBESSE et al., 2006).

Os valores superiores observados nos índices de E\% e IVE (tabela 1) nos tratamentos que apresentavam terra de barranco (S2, S3 e S4) como principal material para as sementes de cajuzinhoarbóreo-do-cerrado, confirmam que a capacidade de retenção de umidade do substrato representa um fator de influência à germinação das sementes. A terra de barranco comumente encontrada nos solos antigos e bem drenados do Cerrado conferiu boas condições de armazenamento de água e por conseguinte a germinação das sementes.

Os dados de emergência obtidos nesta pesquisa ainda se assemelham aos resultados de Borges et al. (2012) em estudo com cajuzinho-arbóreo-do-cerrado de diferentes regiões, que observou porcentuais de emergência de 56,67\% e 86,67\% em Calcilândia e Vila Propício (GO), respectivamente, enquanto Borges et al. (2013), avaliando a taxa de germinação de sementes de cajuzinho-arbóreo-do-cerrado e o 
Emergência e caracterização biométrica inicial de cajuzinho-arbóreo-do-cerrado (Anacardium othonianum Rizz.) em diferentes temperaturas e substratos

PINTO, A. F. J.; CARDOSO, M. T. R.; SILVA, C. L. T.; PEREIRA, W. J.; ISSA, C. G. C.; SOUZA, E. R. B.; VIEIRA, M. C.; SIQUEIRA, A. P. S.

desenvolvimento inicial das plantas em campo, obteve uma germinação média de $78 \%$.

A importância de se avaliar o número de par de folhas, está no fato da folha ser responsável pela fotossíntese e por grande parte da respiração da planta, então, assim como a quantidade de folhas, o tamanho destas folhas também é fator importante para uma muda de qualidade. No presente trabalho, as plantas cultivadas no substrato S4 apresentaram o maior número de par de folhas (tabela 2; figura 2), assim, mudas oriundas deste substrato tende a apresentar plantas mais eficientes para realizar a fotossíntese.

A altura da parte aérea avaliada isoladamente, é uma variável para expressar a qualidade das mudas, entretanto, é importante combinar essa variável com outras como o diâmetro do coleto, pesos e relação das raízes e da parte aérea (GOMES et al., 2002; CALDEIRA et al., 2008). A maior altura de plantas observada nas plantas cultivadas no substrato $\mathrm{S} 4$ a uma temperatura de $42^{\circ} \mathrm{C}$ (tabela 2; figura 3) deve-se ao substrato ter como material base a terra de barranco. Ainda foi possível verificar que ao fim dos 40 dias, algumas mudas de cajuzinho-arbóreo-do-cerrado, apresentavam crescimento levemente estiolado. Este fato ocorreu pela exposição da muda a um período prolongado a temperatura de $42^{\circ} \mathrm{C}$.

O diâmetro do caule também é uma variável importante a ser avaliada, pois, o crescimento do diâmetro do caule é considerado uma medida estacionária de crescimento secundário o que indica vigor da planta (MESQUITA et al., 2004). As mudas cultivadas no substrato S4 também apresentaram resultados superiores de diâmetro, indicando que as mudas provenientes deste substrato são mais vigorosas em comparação aos demais tratamentos, e, portanto, resultaram em plantas mais resistentes aos fatores ambientais. A altura da planta e o diâmetro do caule são indicadores importantes da capacidade de sobrevivência da muda, visto que refletem o desenvolvimento radicular e a capacidade de adaptação às condições adversas de campo (PRATES et al., 2012).

A maior largura de folhas foi encontrada nas mudas cultivadas no substrato S2 (tabela 2), enquanto, as mudas cultivadas no substrato $\mathrm{S} 4$ foram as que apresentaram os maiores comprimento de folhas. Plantas com folhas mais largas e compridas tendem a gerar uma copa mais exuberante, além de, possuírem maior área foliar para a captação de energia luminosa no processo de fotossíntese.

Os resultados superiores de comprimento de raiz verificados no substrato S4 (tabela 2; figura 5), deve-se a presença da casca de arroz. A casca de arroz apresenta baixa capacidade de retenção de água, drenagem rápida e eficiente, proporcionando boa oxigenação para as raízes, elevado espaço de aeração ao substrato, resistência à decomposição, relativa estabilidade de estrutura, baixa densidade e pH próximo à neutralidade (MELLO, 2006; STEFFEN et al., 2010). Entretanto, deve-se ficar atento a esta caraterísticas, pois algumas espécies arbóreas do cerrado possuem maior crescimento do sistema radicular em relação à parte aérea, devendo ter o cuidado de fazer o transplante das mudas antes que haja prejuízo da expansão do sistema radicular (SOUZA et al., 2010).

Quando se trabalha com temperatura, nem sempre a muda com maior altura de planta e diâmetro de caule, apresentará as maiores massas fresca e seca, como ocorreu para as mudas oriundas do substrato S2 e S4 (tabela 3). O substrato S2 proporcionou mudas de cajuzinho-arbóreo-do-cerrado com os maiores 
valores de massas fresca e seca $(3,34$ e $0,33 \mathrm{~g}$, respectivamente), enquanto, a maior altura de plantas e diâmetro de caule foram verificados nas plantas do substrato S4 (tabela 2).

A importância de se avaliar as massas fresca e seca é que o rendimento da cultura depende da produção total de biomassa e da distribuição da matéria seca entre partes produtivas e não produtivas das plantas, sendo necessário entender as fontes de variação e controle que contribuem para as diferenças na distribuição (HOLE et al., 1983). Ademais, a produção de biomassa apresenta relação direta com a quantidade de radiação fotossinteticamente absorvida pelo dossel da planta (MONTEITH, 1972; STRASSBURGER et al., 2011). Além disso, para análises de crescimento é necessário obter a medida do aumento do acúmulo de matéria orgânica, considerando os pesos das partes secas da planta (frutos, caule, folhas e outros) (FONTES et al., 2005).

\section{CONCLUSÕES}

O substrato S4 (terra de barranco + vermiculita + palha de arroz) proporcionou as melhores condições de emergência e desenvolvimento para mudas cajuzinho-arbóreo-do-cerrado, por possuir características como: aeração, drenagem, retenção de água e disponibilidade balanceada de nutrientes. A temperatura ótima para o cultivo de cajuzinho-arbóreo-do-cerrado foi de $30^{\circ} \mathrm{C}$ com melhores porcentagem de germinação e velocidade de germinação.

\section{REFERÊNCIAS}

AGOSTINI-COSTA, T. S.; FARIA, J. P.; NAVES, R. V.; VIEIRA, R. F.. Cajus do cerrado. In: VIEIRA, R. F.; AGOSTINI-COSTA T. S.; SILVA, D. B.; FERREIRA, F. R.; SANOC, S. M.. Frutas nativas da Região Centro-Oeste do Brasil. Brasília: EMBRAPA, 2006. p.135-152.

ALVES M. S. O.; ALVES A. M.; NAVES M. M. V.. Compostos bioativos e atividade antioxidante de pseudofrutos de caju arbóreo do Cerrado. Revista do Instituto Adolfo Lutz, São Paulo, v.72, n.4, p.327-331, 2013. DOI: https://doi.org/10.18241/0073-98552013721582

ALVES, M. T. R.; MACHADO, K. B.; FERREIRA, M. E.; VIERIRA, L. C. G.; NABOUT, J. C.. Um instantâneo das características limnológicas em lagos de várzea tropicais: a influência relativa do clima e do uso da terra. Acta Limnologica Brasiliensia, Rio Claro, v.31, n.10, 2019. DOI: http://doi.org/10.1590/s2179-975x7916

ANDRADE, A. C. S.; SOUZA, A. F.; RAMOS, F. N.; PEREIRA, T. S.; CRUZ, A. P. M.. Germinação de sementes de jenipapo: temperatura, substrato e morfologia do desenvolvimento pós-seminal. Pesquisa Agropecuária Brasileira, Brasília, v.35, n.3, p.609-615, 2000. DOI: https://doi.org/10.1590/s0100$\underline{204 \times 2000000300017}$

APARECIDA-DIAS, M.; LOPES, J. C.; SOUZA NETO, J. D.; HEBERLE, E.. Influência da temperatura e substrato na germinação de sementes de jabuticabeira (Myrciaria cauliflora Berg.). Idesia, v.29, n.1, p.23-27, 2011. DOI: https://doi.org/10.4067/s0718-34292011000100004
ARAÚJO-NETO, J. C.; AGUIAR, I. B.; FERREIRA, V. M.. Efeito da temperatura e da luz na germinação de sementes de Acacia polyphylla DC. Brazilian Journal of Botany, São Paulo, v.26, n.2, p.249-256, 2003.

DOI: https://doi.org/10.1590/s0100-84042003000200013

ASSIS, K. C.; PEREIRA, F. D.; CABRAL, J. S. R.; SILVA, F. G.; SILVA, J. W.; SANTOS, S. C.. dos. Cultivo in vitro de Anacardium othonianum Rizz.: efeito da variação das concentrações dos sais e volumes do meio de cultura. Acta Scientiarum Agronomy, Maringá, v.34, n.1, p.77-83, 2012. DOI: http://doi.org/10.1590/S1807-86212012000100011

BESSA, L. A.; SILVA, F. G.; MOREIRA, M. A.; TEODORO, J. P. R.; SOARES, F. A. L.. Growth and nutrient accumulation of Anacardium othonianum Rizz. seedlings grown in nutrient solution. Chilean Journal of Agricultural Research, Chillán, v.73, n.3, p.301-308, 2013. DOI:

http://doi.org/10.4067/S0718-58392013000300014

BEUCHLE, R.; GRECCHI, R. C.; SHIMABUKURO, Y. E.; SELIGER, R.; EVA, H. D.; SANO, E.; ACHARD, F.. Land cover changes in the Brazilian Cerrado and Caatinga biomes from 1990 to 2010 based on a systematic remote sensing sampling approach. Applied Geography, Oxford, v.58, p.116-127, 2015. DOI: https://doi.org/10.1016/j.apgeog.2015.01.017

BEWLEY, J. D.; BLACK, M.. Seeds: physiology of development and germination. Nova lorque: Plenum Press, 1994. DOI: http://doi.org/10.1007/978-1-4899-1002-8

BORGES, R. T.. Caracterização do ambiente de ocorrência 
natural, fruto e pseudofruto de caju arbóreo do cerrado (Anacardium othonianum): fenologia e implantação de coleção na EA/UFG. Dissertação (Mestrado em Agronomia) Universidade Federal de Goiás, Goiânia, 2012.

BORGES, R. T.; SOUZA, E. R. B.; NAVES, R. V.; BELO, A. P. M.; CAMILO, Y. M. V.. Produção de mudas, coleções e estabelecimento de caju arbóreo do cerrado (Anacardium othonianum) no estado de Goiás. Enciclopédia Biosfera, Goiânia, v.9, n.17, p.2107-2120, 2013.

CAETANO, G. S.; SOUSA, K. A.; RESENDE, O.; SALES, J. F.; COSTA, L. M.. Higroscopicidade de sementes de caju-deárvore-do-cerrado. Pesquisa Agropecuária Tropical, Goiânia, v.42, n.4, p.437-445, 2012. DOI:

https://doi.org/10.1590/s1983-40632012000400012

CALDEIRA, M. V. W.; ROSA, G. N.; FENILLI, T. A. B.; HARBS, R. M. P.. Composto orgânico na produção de mudas de aroeiravermelha. Scientia Agraria, Curitiba, v.9, n.1, p.27-33, 2008. DOI: http://doi.org/10.5380/rsa.v9i1.9898

DAMIANI, C.; BOAS, E. V. B. V.; ASQUIERI, E. R.; LAGE, M. E.; OLIVEIRA, R. A.; SILVA, F. A.; PINTO, D. M.; RODRIGUES, L. J.; SILVA, E. P.; PAULA, N. R. F.. Characterization of fruits from the savanna: araça (Psidium guinnensis Sw.) and marolo (Annona crassiflora Mart.). Ciência e Tecnologia de Alimentos, Campinas, v.31, n.3, p.723-729, 2011. DOI: http://doi.org/10.1590/S0101-20612011000300026

DORNELLES, P.; SILVA, F. G.; MOTA, C. S.; SANTANA, J. G. Production and quality of Anacardium othonianum Rizz. seedlings grown in different substrates. Revista Brasileira de Fruticultura, Jaboticabal, v.36 n.2, p.479-486, 2014. DOI: http://doi.org/10.1590/0100-2945-144/13

DOUSSEAU, S.; ALVARENGA, A. A. D.; GUIMARÃES, R. M.; LARA, T. S.; CUSTÓDIO, T. N.; CHAVES, I. D. S.. Ecofisiologia da germinação de sementes de Campomanesia pubescens. Ciência Rural, Santa Maria, v.41, n.8, p.1362-1368, 2011. DOI: https://doi.org/10.1590/s0103-84782011000800011

DRESCH, D. M.; SCALON, S. P. Q.; MASETTO, T. E.; VIEIRA, M. C.. Germinação de sementes de Campomanesia adamantium (Camb.) O. Berg em diferentes temperaturas e umidades do substrato. Scientia Forestalis, Piracicaba, v.40, n.94, p.223-229, 2012.

FACHINELLO, J. C.; HOFFMAN, A.; NACHTIGAL, J. C.; KERSTEN, E.; FORTES, G. R. L.. Propagação de plantas frutíferas de clima temperado. 2 ed. Pelotas: EDUFPel, 1995.

FACHINELLO, J. C.; HOFFMANN, A.; NACHTIGAL, J. C.. Formas de propagação de plantas frutíferas. In: FACHINELLO, J. C.; HOFFMANN, A.; NACHTIGAL, J. C.. Propagação de plantas frutíferas. Brasília: EMBRAPA, 2005.

FERRAZ, M. V.; CENTURION, J. F.; BEUTLER, A. N.. Caracterização física e química de alguns substratos comerciais. Acta Scientiarum Agronomy, Maringá, v.27, n.2, p.209-214, 2005. DOI:

https://doi.org/10.4025/actasciagron.v27i2.1483

FERREIRA, D. F.. Análise estatística por meio do Sisvar (Sistema para análise de variância) para Windows versão 4.0.
In: REUNIÃO ANUAL DA REGIÃO BRASILEIRA DA SOCIEDADE INTERNACIONAL DE BIOMETRIA, 45. Anais. São Carlos: UFSCar, 2000.

FONTES, P. C. R.; DIAS, E. N.; SILVA, D. J. H.. Dinâmica do crescimento, distribuição de matéria seca na planta e produção de pimentão em ambiente protegido. Horticultura Brasileira, Brasília, v.23, n.1, p.94-99, 2005. DOI: https://doi.org/10.1590/s0102-05362005000100020

GOMES, J. M.; COUTO, L.; LEITE H. G.; XAVIER, A.; GARCIA, S. L. R.. Parâmetros morfológicos na avaliação da qualidade de mudas de Eucalyptus grandis. Árvore, v.26, n.6, p.655-664, 2002. DOI: https://doi.org/10.1590/s0100$\underline{67622002000600002}$

GOMES, J. P.; OLIVEIRA, L. M.; FERREIRA, P. L.; BATISTA, F.. Substratos e temperaturas para teste de germinação em sementes de Myrtaceae. Ciência Florestal, Santa Maria, v.26, n.4, p.285-293, 2016. DOI:

http://doi.org/10.5902/1980509821120

HOLE, C. C.; BARNES, A.; THOMAS, T. H.; SCOTT, P. A.; RANKIN, W. E. F.. Dry matter distribution between the shoot and storage root of carrot (Daucus carota L.). I. Comparison of varieties. Annual Botany, v.51, n.2, p.175-187. 1983. DOI: http://doi.org/10.1093/oxfordjournals.aob.a086456

KANASHIRO, S.. Efeito de diferentes substratos na produção da espécie Aechemea fasciata (Lindley) Baker em vasos. Dissertação (Mestrado em Fitotecnia) - Universidade de São Paulo, Piracicaba, 1999.

LATRUBESSE, E. M.; STEVEAUX, J. C.. Características físicobióticas e problemas ambientais associados à planície aluvial do Rio Araguaia, Brasil Central. Geociências, v.5, n.1, p.6573, 2006.

MAYER, A. M.; POLJAKOFF-MAYBER, A.. The germination of seeds. 3 ed. Nova lorque: Elsevier, 1982. DOI: https://doi.org/10.1016/C2013-0-03635-3

MELLO, R. P.. Consumo de água do lírio asiático em vaso com diferentes substratos. Dissertação (Mestrado em Engenharia Agrícola) - Universidade Federal de Santa Maria, Santa Maria, 2006

MESQUITA, R. C. M.; PARENTE, J. I. G.; MONTENEGRO, A. A. T.; MELO, F. I. O.; PINHO, J. L. N.; CAVALCANTI JÚNIOR, A. T. Influência de regimes hídricos na fenologia do crescimento de clones e progênies de cajueiro precoce e comum nos primeiro vinte meses. Ciência Agronômica, Fortaleza, v.35, n.1, p.96-103, 2004.

MONTEITH, J. L.. Solar Radiation and Productivity in Tropical Ecosystems. The Journal of Applied Ecology, Oxford, v.9, n.3, p.747-766, 1972. DOI: https://doi.org/10.2307/2401901

MORZELLE, M. C.; BACHIEGA, P.; SOUZA, E. C.; BOAS, E. V. B. V.; LAMOUNIER, M. L.. Caracterização química e física de frutos de curriola, gabiroba e murici provenientes do Cerrado brasileiro. Revista Brasileira de Fruticultura, Jaboticabal, v.37, n.1, p.96-103, 2015. DOI: http://doi.org/10.1590/0100-2945-036/14

OLIVEIRA, L. M.; CARVALHO, M. L. M.; SILVA, T. T. A.; 
BORGES, D. I.. Temperatura e regime de luz na germinação de sementes de Tabebuia impetiginosa (Martius ex A. P. de Candolle) Standley e T. serratifolia Vahl Nich. - Bignoniaceae. Ciência Agrotécnica, Lavras, v.29, n.3, p.642-648, 2005. DOI: http://doi.org/10.1590/S1413-70542005000300020

OLIVEIRA, P. T. S.; NEARING, M. A.; MORAN, M. S.; GOODRICH, D. C.; WENDLAND, E.; GUPTA, H. V.. Trends in water balance components across the Brazilian Cerrado. Water Resources Research, Washington, v.50, n.9, p.71007114, 2014. DOI: http://doi.org/10.1002/2013WR015202

PACHECO, M. V.; MATOS, V. P.; FERREIRA, R. L. C.; FELICIANO, A. L. P.; PINTO, K. M. S.. Efeito de temperaturas e substratos na germinação de sementes de Myracrodruon urundeuva fr. All. (Anacardiaceae). Árvore, Viçosa, v.30, n.3, p.359-367, 2006. DOI: https://doi.org/10.1590/s0100$\underline{67622006000300006}$

PRATES, F. B. S.; LUCAS, C. S. G.; SAMPAIO, R. A.; JÚNIO, D. S. B.; FERNANDES, L. A.; JUNIO, G. R. Z.. Crescimento de mudas de pinhão-manso em resposta a adubação com superfosfato simples e pó-de-rocha. Ciência Agronômica, Fortaleza, v.43, n.2, p.207-213, 2012. DOI: https://doi.org/10.1590/s180666902012000200001

RIBEIRO, S. C.; FEHRMANN, L.; SOARES, C. P. B.; JACOVINE, L. A. G.; KLEINN, C.; GASPAR, R. de O.. Above and belowground biomass in a Brazilian Cerrado. Forest Ecology and Management, Amsterdã, v.262, n.3, p.491-499, 2011. DoI: http://doi.org/10.1016/j.foreco.2011.04.017

SANO, E. E.; ROSA, R.; BRITO, J. L. S.; FERREIRA, L. G.. Land cover mapping of the tropical savanna region in Brazil. Environmental Monitoring and Assessment, Dordrecht, v.166, n.4, p.113-124, 2010. DOI: http://doi.org10.1007/s10661-009-0988-4

SCHIASSI, M. C. E. V.; SOUZA, V. R.; LAGO, A. M. T.; CAMPOS, L. G.; QUEIROZ, F.. Fruits from the Brazilian Cerrado region: Physico-chemical characterization, bioactive compounds, antioxidant activities, and sensory evaluation. Food Chemistry, Londres, v.245, p.305-311, 2018. DOI: https://doi.org/10.1016/i.foodchem.2017.10.104

SEO, M.; NAMBARA, E.; CHOI, G.; YAMAGUCHI, S. Interaction of light and hormone signals in germinating seeds. Plant Molecular Biology, Dordrecht, v.69, n.4, p.463472, 2009. DOI: https://doi.org/10.1007/s11103-008-9429-y
SILVA, E. A.; MARUYAMA, W. I.; OLIVEIRA, A. C.; BARDIVIESSO, D. M.. Efeito de diferentes substratos na produção de mudas de mangabeira (Hancornia speciosa). Revista Brasileira de Fruticultura, Jaboticabal, v.31, n.3, p.925-929, 2009. DOI: https://doi.org/10.1590/s0100$\underline{29452009000300043}$

SILVA, J. C. F.; SOUZA, I. A.; CONCEIÇÃO, D. R.; CORDEIRO, C. A. S.; MACHADO, A. S.; RIOS, A. D. F.. Desenvolvimento inicial do cajuzinho do cerrado. Ipê Agronomic Journal, Goianésia, v.2, n.1, p.23-30, 2018.

SILVA, L. A.; SALES, J. F.; NEVES, J. M. G.; OLIVEIRA, H.; SILVA, G. P.. Radiographic image analysis of Anacardium othonianum Rizz (Anacardiaceae) achenes subjected to desiccation. Acta Scientiarum Agronomy, Maringá, v.39, n.2, p.235-244, 2017. DOI: http://doi.org/10.4025/actasciagron.v39i2.32484

SOUZA, E. R. B.; ALMEIDA, K. S.; CAMILO, Y. M. V.; MARRA, G. E. R.; SELEGUINI, A.; NAVES, R. V.; CORRÊA, G. C.. Avaliação agronômica do cajuzinho-do-cerrado (Anacardium othonianum Rizz.) em Goiás. In: ENCONTRO NACIONAL DE SUBSTRATOS PARA PLANTAS. 7. Anais. Goiânia: UFG, 2010.

STEFFEN, G. P. K.; ANTONIOLLI, Z. I.; STEFFEN, R. B.; MACHADO, R. G.. Casca de arroz e esterco bovino como substratos para a multiplicação de minhocas e produção de mudas de tomate e alface. Acta Zoológica Mexicana, Xalapa, v.26, n.2, p.333-343, 2010. DOI:

https://doi.org/10.21829/azm.2010.262898

STOCKMAN, A. L.; BRANCALION, P. H. S.; NOVEMBRE, A. D. L.; CHAMMA, H. M. C. P.. Sementes de ipê-branco (Tabebuia roseo-alba (Ridl.) Sand. - Bignoniaceae): temperatura e substrato para o teste de germinação. Revista Brasileira de Sementes, Brasília, v.29, n.3, p.139-143, 2007. DOI: https://doi.org/10.1590/s0101-31222007000300016

STRASSBURGER, A. S.; PEIL, R. M. N.; FONSECA, L. A.; AUMONDE, T. Z.; MAUCH, C. R.. Dinâmica de crescimento da abobrinha italiana em duas estações de cultivo. Acta Scientiarum Agronomy, Maringá, v.33, n.2, p.283-289, 2011. DOI: http://doi.org/10.4025/actasciagron.v33i2.5952

TOSCHI, T. G.; CABONI, M. F.; PENAZZI, G.; LERCKER, G.; CAPELLA, P.. A study on cashew nut oil composition. Journal of the American Oil Chemist's Society, Champaign, v.70, n.10, p.1017-1020, 1993. DOI: https://doi.org/10.1007/BF02543029

A CBPC - Companhia Brasileira de Produção Científica (CNPJ: 11.221.422/0001-03) detém os direitos materiais desta publicação. Os direitos referem-se à publicação do trabalho em qualquer parte do mundo, incluindo os direitos às renovações, expansões e disseminações da contribuição, bem como outros direitos subsidiários. Todos os trabalhos publicados eletronicamente poderão posteriormente ser publicados em coletâneas impressas sob coordenação da Sustenere Publishing, da Companhia Brasileira de Produção Científica e seus parceiros autorizados. Os (as) autores (as) preservam os direitos autorais, mas não têm permissão para a publicação da contribuição em outro meio, impresso ou digital, em português ou em tradução. 